\title{
Magnetic Plasmon Resonance
}

\author{
Andrey K. Sarychev \\ Ethertronics Inc., San Diego, CA 92121 \\ Gennady Shvets \\ Department of Physics, \\ The University of Texas at Austin, Austin, TX 78712 \\ Vladimir M. Shalaev \\ School of Electrical and Computer Engineering, \\ Purdue University, West Lafayette, IN 47907
}

January 12, 2006

\begin{abstract}
It is demonstrated that metallic horseshoe-shaped (also referred to as u-shaped) nanostructures can exhibit a magnetic resonance in the optical spectral range. This magnetic plasmon resonance is distinct from the purely geometric LC resonance occurring in perfectly conducting split rings because the plasmonic nature of the metal plays the dominant role. Similarly to the electrical surface plasmon resonance, the magnetic plasmon resonance is determined primarily by the metal properties and nanostructure geometry rather than by the ratio of the wavelength and the structure's size. Magnetic plasmon resonance occurs in nanostructures much smaller in size than the optical wavelength. Electromagnetic properties of periodically assembled horseshoe-shaped nanostructures are investigated, and the close proximity of the electrical and magnetic plasmon resonances is exploited in designing a negative index meta-material. Close to the magnetic plasmon resonance frequency both magnetic permeability $\mu$ and electric permittivity $\varepsilon$ can become negative, paving the way for the development of sub-wavelength negative index materials in the optical range.
\end{abstract}




\section{Introduction}

Extending the range of electromagnetic properties of naturally occurring materials motivates the development of artificial (or meta-) materials. For example, it has recently been demonstrated that meta-materials may exhibit such interesting properties as negative dielectric permittivity $\varepsilon<0$ (see, for example [1], [2]), negative magnetic permeability $\mu<0$ [3], and even both $[4,5]$. The double-negative case of $\varepsilon<0$ and $\mu<0$ (often referred to as the left-handed and negative refractive regime) is particularly interesting because of the possibility of making a "perfect" lens with sub-wavelength spatial resolution [6]. In addition to the super resolution, unusual and sometimes counter-intuitive properties of negative index materials (NIMs), which also referred to as left-handed materials (LHMs), make them very promising for applications in resonators, waveguides and other microwave and optical elements $[7,8,9,10]$.

Negative refraction has been convincingly demonstrated in the microwave regime $[5,8,9,11]$. For microwave NIMs, artificial magnetic elements (providing $\mu<0$ ) are the split-ring resonators or the swiss roll structures. In the microwave part of the spectrum, metals can be considered as perfect conductors because the skin depth is much smaller than the metallic feature size. The strong magnetic response is achieved by operating in the vicinity of the LC resonance of the split ring $[3,12]$. The same technique of obtaining $\mu<0$ using split rings was recently extended to mid-IR [12] by scaling down the dimensions of the split rings. In the microwave, as well as (to a lesser degree) in the mid-IR part of the spectrum, metals can be approximated as perfect conductors because the skin depth is much smaller than the feature size of the structure. Therefore, the frequencies of the LC resonances are determined entirely by the split ring geometry and size but not by the electromagnetic properties of the metal. In accordance with this, the ring response is (resonantly) enhanced at some particular ratio of the radiation wavelength and the structure size. Thus we refer to the LC resonances of perfectly conducting metallic structures as geometric LC (GLC) resonances.

The situation drastically changes in the optical part of the spectrum, where thin (sub-wavelength) metal components behave very differently when their sizes become less than the skin depth. For example, the electrical surface plasmon resonance (SPR) occurs in the optical and near-IR parts of the spectrum due to collective electron oscillations in metal structures. Many important plasmon-enhanced optical phenomena and applications of metal 
nano-composites are based on the electrical SPR (see, for example, [13]). Plasmonic nature of the electromagnetic response in metals for optical/midIR frequencies is the main reason why the original methodology of GLC resonances in microwave/mid-IR spectral range is not extendable to higher frequencies.

For the optical range, NIMs with a negative refractive-index have been for the first time demonstrated in the experiment [14] where the authors observed the real part of the refractive index $n=-0.3$ at the telecommunication wavelength of $1.5 \mu \mathrm{m}$. In that report the authors experimentally verified their early theoretical prediction for negative refraction in an array of parallel metal nanorods [15]. Note that the losses become progressively important with increasing frequency toward the optic band. Moreover, the elementary cell of the resulting structure is on order of the wavelength. Making a true NIM requires the cell size to be less than $\lambda / 2$. Therefore, miniaturization of the cell size is of major interest, and can be accomplished, for example, by utilizing plasmonic effects $[16,17,18,19]$.

It is also necessary to take into account from the beginning that dielectric permittivity $\varepsilon=\varepsilon^{\prime}+i \varepsilon^{\prime \prime}$ and magnetic permeability $\mu=\mu^{\prime}+i \mu^{\prime \prime}$ are complex values. The structure that exhibits the negative real dielectric permittivity $\varepsilon^{\prime} \approx-0.7$ and negative real of magnetic permeability $\mu^{\prime} \approx-0.3$ in green light $(\lambda \approx 0.5 \mu \mathrm{m})$ has been investigated in Ref. [20]. However rather large imaginary components ( $\mu^{\prime \prime} \approx 1.0$ at the resonance) has not allowed the observation of the negative refraction.

Thus the demonstration of a negative index meta-material in the regime where plasmonic effects are important remains elusive. Plasmonic effects must be correctly accounted for to design a meta-material with optical magnetism. Below we show that specially arranged metal nanoparticles can support, along with the electrical SPR, magnetic plasmon resonance (MPR). The MPR's resonance frequency $\omega_{r}$ can be made independent of the absolute characteristic structure size $a$ and $\lambda / a \equiv 2 \pi c / \omega a$. The only defining parameters are the plasmonic permittivity $\varepsilon_{m}(\omega)$ and the structure geometry. Such structures act as optical nanoantennas by concentrating large electric and magnetic energies on the nanoscale at the optical frequencies. The magnetic response is characterized by the magnetic polarizability $\alpha_{M}$ with the resonant behavior similar to the electric SPR polarizability $\alpha_{E}$ : real part of $\alpha_{M}$ changes the sign near the resonance and becomes negative for $\omega>\omega_{r}$, as required for negative index meta-materials. We show that the electrostatic resonances must replace (or strongly modify) GLC resonances in the 
optical/mid-IR range if a strong magnetic response is desired.

The idea of using electrostatic resonances for inducing optical magnetism is relatively recent. For example, electrostatic resonances of periodic plasmonic nanostructures have been recently employed to induce magnetic properties due to close proximity of adjacent nanowires [16, 17]. Higher multipole electrostatic resonances were shown [18] to hybridize in such a way as to induce magnetic moments in individual nanowires. Strong electrostatic resonances of regularly shaped nanoparticles (including nanospheres and nanowires) occur for $-2<\varepsilon_{m}^{\prime}<-1$. The resistive damping characterized by the ratio $\varepsilon_{m}^{\prime \prime} / \varepsilon_{m}^{\prime}$ of the imaginary and real parts of the dielectric permittivity of a metal is fairly strong for those frequencies corresponding to $\left|\varepsilon_{m}^{\prime}\right| \sim 1$. However, $\varepsilon_{m}^{\prime \prime} / \varepsilon_{m}^{\prime}$ is known to decrease for $\left|\varepsilon_{m}\right| \gg 1$. Therefore, there is a considerable incentive to design nanostructures exhibiting resonances for $\varepsilon_{m}^{\prime} \ll-1$. Such horseshoe-shaped structures, first suggested in Ref. [21] are described below. Spectrally, these structures support strong magnetic moments at the frequencies higher than the microwave/mid-IR frequencies supported by the traditional split ring resonators (see Refs. [3, 12] for details) and lower than the ultraviolet frequencies supported by the sub-wavelength plasmonic crystals described in Refs. $[17,18]$. Conceptually, the horseshoeshaped structures described here are distinct from the earlier low frequency structures because they are not relying on the GLC resonance for producing a strong magnetic response because plasmonic properties of the metal are very important when the sizes are small and the operational frequencies are high. Below we present a three-dimensional theory of the magnetic resonance in a plasmonic structure and related two-dimensional numerical simulations.

Specifically, here we develop a comprehensive theory and perform detailed numerical simulations for negative index meta-materials based on horseshoeshaped structures. The possibility of optical magnetism in such structures was first theoretically predicted [21] and recently experimentally verified [22]. Closely related split-ring resonator structures were also shown [23] to possess optical magnetism. Here we demonstrate that such horseshoe-shaped structures may have negative dielectric permittivity, in parallel with negative magnetic permeability, and thus they can be used for building a metamaterial with a negative- refractive index. 


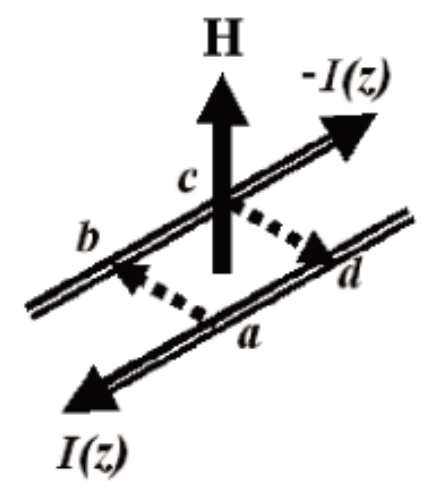

Figure 1: Currents in two parallel metal wires excited by external magnetic field H. Displacement currents, "closing"the circuit, are shown by dashed lines.

\section{Analytical theory of magnetic plasmon res- onances}

We consider first a pair of parallel metallic rods. The external magnetic field excites the electric current in the pair of the rods as shown in Fig. 1. The magnetic moment associated with the circular current flowing in the rods results in the magnetic response of the system. Suppose that an external magnetic field $\mathbf{H}=\left\{0, H_{0} \exp (-i \omega t), 0\right\}$ is applied perpendicular to the plane of the pair (We suppose that magnetic field is along $y$ axis and the rods are in $\{x, z\}$ plane). The circular current $I(z)$ excited by the time-varying magnetic field flows in the opposite directions in the nanowires as shown in Fig. 1. The displacement currents flowing between the nanowires close the circuit. We introduce the "potential drop" $U(z)=\int_{a}^{b} \mathbf{E} d \mathbf{l}$ between the pair where the integration is along the line $\{a(z), b(z)\}$. To find the current $I(z)$, we integrate the Faraday's Law curl $\mathbf{E}=i k\left(\mathbf{H}_{0}+\mathbf{H}_{i n}\right)$ over the contour $\{a, b, c, d\}$ in Fig. 1, where $k=\omega / c$ is a wave vector, and $\mathbf{H}_{i n}=$ curl $\mathbf{A}$ is the magnetic field induced by the current. It is assumed that the nanowire length $2 a$ is much larger than the distance $d$ between the nanowires and the radius $b$ of a nanowire. We also assume that $k d \ll 1$. Under these assumptions, the vector potential $\mathbf{A}$ is primarily directed along the nanowires ( $z$ direction). 
The use of the integral form of the Faraday's Law yields

$$
\left(I R-i k 2 A_{z}+d U / d z\right) \Delta z=-i k H_{0} d \Delta z,
$$

where the pair impedance $R \simeq 2 /\left(\sigma \pi b^{2}\right) \approx 8 i /\left(\varepsilon b^{2} \omega\right)$, where $\varepsilon=1+$ $i 4 \pi \sigma / \omega \ll-1$ is the metal complex permittivity, and $\pm I R / 2$ are the electric fields on the surface of the nanowires. Note that the wire resistivity is explicitly taken into account. This sets our calculation apart from the earlier work on the resonances of conducting split ring resonators [24] and conducting stick composites [2] because plasmonic resonances of the wire are now fully accounted for.

Electric field $\mathbf{E}$ can be always presented in terms of the vector potential $\mathbf{A}$ and electric potential $\phi$ as $\mathbf{E}=-\nabla \phi+i k \mathbf{A}$. In the standard Lorentz gage the electric potential $\phi$ equals to $\phi\left(\mathbf{r}_{1}\right)=\int \exp \left(i k r_{12}\right) q\left(\mathbf{r}_{2}\right) / r_{12} d \mathbf{r}_{2}$ and the vector potential $\mathbf{A}\left(\mathbf{r}_{1}\right)=c^{-1} \int \exp \left(i k r_{12}\right) \mathbf{j}\left(\mathbf{r}_{2}\right) / r_{12} d \mathbf{r}_{2}$, where $r_{12}=$ $\left|\mathbf{r}_{1}-\mathbf{r}_{2}\right|, q$ and $\mathbf{j}$ are charge and current density correspondingly. In the case of two long wires the currents flow inside the wires. Correspondingly the vector potential $\mathbf{A}$ has the only component in the direction $z$ of the wires $\mathbf{A}=\left\{0,0, A_{z}\right\}$. Since the vector potential $\mathbf{A}$ is perpendicular to the line $\{a(z), b(z)\}$ the potential drop $U$ in Eq. (1) equals to $U(z)=\int_{a}^{b} \mathbf{E} d \mathbf{l}=\phi_{a}-$ $\phi_{b}$, where $\phi_{a}$ and $\phi_{b}$ are the electric potential in the points $a(z)$ and $b(z)$. We consider the excitation of the antisymmetric mode when the currents in the wires are the same in absolute value but are opposite in the direction (see Fig. 1). Correspondingly the electric charge per unite length $Q(z)=Q_{a}=$ $-Q_{b}$. We assume that the diameter $b$ of the wire is much smaller than the distance $d$ between them and the wire length $2 a \gg d$. Then the potential drop $U(z)$ between the pair estimates as $U(z)=Q(z) / C$, where the inter wire capacitance $C$ is independent of the coordinate $z$ and is estimated in the Appendix as $C \simeq[4 \ln (d / b)]^{-1}$.

The vector potential $A_{z}(z)$ is proportional to the electric current $A_{z}(z)=$ $(L / c) I(z) / 2$, where the wire pair inductance is estimated as $L \simeq 4 \ln (d / b)$ (see Appendix.) Note that both $C$ and $L$ are purely geometric factors that do not depend on the plasmonic nature of the rods. The product $L C$ can be estimated as $L C \simeq 1$. Yet, in the above consideration we never assumed that the wires are made from the perfect metal or from a metal with real conductivity, i.e., imaginary permittivity. Moreover, the two wire nanoantenna has most interesting behavior when metal dielectric constant is real and negative. The plasmonic nature of the metal is accounted for below. 
We substitute $U(z)$ and $A_{z}(z)$ into Eq. (1), taking into account the charge conservation law $d I / d z=i \omega Q(z)$, and obtain a differential equation for the current:

$$
\frac{d^{2} I(z)}{d z^{2}}=-g^{2} I(z)-\frac{C d \omega^{2}}{c} H_{0}
$$

where $-a<z<a, I(-a)=I(a)=0$, and the parameter $g$ is given as

$$
g^{2}=k^{2}\left[L C-8 C\left((k b)^{2} \varepsilon_{m}\right)^{-1}\right] .
$$

The two-wire antenna is resonantly excited when $G=g a=N \pi / 2$, where $N$ is an integer. Note that the material properties of the metal enter the resonant parameter $G$ through the dielectric permittivity $\varepsilon_{m}$. In the context of the wire pair, the earlier discussed GLC resonances [3, 15, 12, 24, 26] correspond to the wire thickness $b$ much larger than the skin depth $\left(k^{2}\left|\varepsilon_{m}\right|\right)^{-1 / 2}$. This approximation, typically valid for microwave and mid-IR frequencies, yields $g=k / \sqrt{L C}$ and the resonant condition $k a=\pi / 2$, also known as the antenna resonance.

Let's consider the opposite ("electrostatic", as explained below) limit of $\left|8 C\left[(k b)^{2} \varepsilon_{m}\right]^{-1}\right| \gg 1$. In the electrostatic regime $G$ depends only on the metal permittivity and the aspect ratio:

$$
G^{2} \simeq-2(a / b)^{2} \ln (d / b) / \varepsilon_{m},
$$

but not on the wavelength and absolute length of the wires. Sharp resonance in Eq. (2) requires that $G^{2}$ be positive, possibly with a very small imaginary part. Indeed, for IR/visible frequencies $\varepsilon_{m}$ is negative (with a smaller imaginary part) for typical (Ag, $\mathrm{Au}$, etc.) low loss metals. Metal dielectric constant $\varepsilon_{m}$ can be approximated by the Drude formula:

$$
\varepsilon_{m}(\omega) \cong \varepsilon_{b}-\left(\omega_{p} / \omega\right)^{2} /\left(1-i \omega_{\tau} / \omega\right),
$$

where $\varepsilon_{b}$ is a "polarization" constant, $\omega_{p}$ is the plasma frequency, and $\omega_{\tau}=$ $1 / \tau$ is the relaxation rate. For considered here silver nanoantennas the constant $\varepsilon_{b}$ approximates as $\varepsilon_{b} \approx 5$, the plasma frequency $\omega_{p} \approx 9.1$ and the relaxation rate $\omega_{\tau} \approx 0.02[28]$. For example, at $\lambda=1.5 \mu \mathrm{m}$ the silver dielectric constant estimates as $\varepsilon_{m}^{\prime} \approx-120$ and $\varepsilon_{m}^{\prime \prime} /\left|\varepsilon_{m}\right| \approx 0.025$.

We consider now the electric field in the system of two conducting rods still assuming the electrostatic limit when the propagation constant $G$ is 
given by Eq. (4). The electric charge $Q(z)$ and the current $I(z)(Q(z)=$ $\left.(i \omega)^{-1} d I(z) / d z\right)$ are given by solution of Eq. (2)

$$
\begin{aligned}
& Q(z)=Q_{0} \frac{\sin (G z / a)}{\cos G} \\
& I(z)=i \frac{Q_{0} a \omega}{G}\left(1-\frac{\cos (G z / a)}{\cos G}\right),
\end{aligned}
$$

where $Q_{0}=i b d k H_{0} \sqrt{-\varepsilon_{m}} /\left[4 \sqrt{2} \ln ^{3 / 2}(d / b)\right]$. Using the Lorentz gauge we can write the equation for the electric potential

$$
\phi(r)=\int q\left(\mathbf{r}_{1}\right) \frac{\exp \left(i k R_{1}\right)}{R_{1}} d \mathbf{r}_{1}-\int q\left(\mathbf{r}_{2}\right) \frac{\exp \left(i k R_{2}\right)}{R_{2}} d \mathbf{r}_{2},
$$

where $q\left(r_{1}\right)$ and $q\left(r_{2}\right)$ are electric charges distributed over the surface of the rods 1 and $2, R_{1}=\left|\mathbf{r}-\mathbf{r}_{1}\right|, R_{2}=\left|\mathbf{r}-\mathbf{r}_{2}\right|$, and the integration goes over the rods 1 and 2 . We consider the electric field between the rods, i.e., in $\{z, x\}$ plane (see Fig. 1) and assume that $|x| \ll a,|z|<a$, and the distances to the $\operatorname{rods} d_{1}=|x-d / 2| \gg b$ and $d_{2}=|x+d / 2| \gg b$. Then we can integrate in Eq. (8) over the cross-section of the rod after which it takes the one-dimension form

$$
\phi(x, z)=\int_{-a}^{a} Q\left(z_{1}\right)\left[\frac{\exp \left(i k R_{1}\right)}{R_{1}}-\frac{\exp \left(i k R_{2}\right)}{R_{2}}\right] d z_{1},
$$

where the linear charge density $Q\left(z_{1}\right)$, obtained from $q\left(r_{1}\right)$ by integration over the rod circumference, is given by Eq. (6) $\left[Q\left(z_{1}\right)=-Q\left(z_{2}\right)\right]$, the distances $R_{1}$ and $R_{2}$ take the following form $R_{1}=\sqrt{d_{1}^{2}+\left(z-z_{1}\right)^{2}}, R_{2}=$ $\sqrt{d_{2}^{2}+\left(z-z_{1}\right)^{2}}$. Two terms in the square brackets in Eq. (9) cancel when $\left|z-z_{1}\right|>d$, as it is discussed in the Appendix. Since we assume that $k d \ll 1$ and $d \ll a$ we can put the exponents $\exp \left(i k R_{1}\right) \simeq \exp \left(i k R_{1}\right) \simeq 1$ and extend the integration in Eq. (9) from $z_{1}=-\infty$ to $z_{1}=\infty$. Resulting integral is solved explicitly and we obtain the analytical equation for the electric potential in the system of two nanowires

$$
\phi(x, z)=2 \ln \left|\frac{d / 2+x}{d / 2-x}\right| Q(z),
$$

where $Q(z)$ is given by Eq. (6). Extrapolation of this result to the surface of the wires gives the potential drop $U(z)=\phi(d / 2-b, z)-\phi(-d / 2+b, z)=$ 
$4 \ln (2 d / b) Q(z)$. Thus we obtain that the inter-wire capacitance $C=Q(z) / U(z)$ $=4 \ln (d / b)$ is a constant, which is independent of the coordinate $z$, in agreement with estimate done in the Appendix.

The vector potential $\mathbf{A}=\{0,0, A\}$ is calculated in a similar way

$$
\begin{aligned}
A(x, z) & \simeq \frac{1}{c} \int_{-a}^{a} I\left(z_{1}\right)\left[\frac{\exp \left(i k R_{1}\right)}{R_{1}}-\frac{\exp \left(i k R_{2}\right)}{R_{2}}\right] d z_{1} \\
& \simeq \frac{1}{c} \int_{-\infty}^{\infty} I\left(z_{1}\right)\left[\frac{1}{R_{1}}-\frac{1}{R_{2}}\right] d z_{1} \simeq \frac{2}{c} \ln \left|\frac{d / 2+x}{d / 2-x}\right| I(z) .
\end{aligned}
$$

where the electric current is given by Eq. (7). Extrapolating to the vector potential $A$ to the surface of the first wire $(x=d / 2-b)$ we obtain $2 c A=L I$, where the inter-wire inductance $L$ equals to $L \simeq 4 \ln (d / b)$. The inductance $L$ is also independent of the coordinate $z$ in agreement with the Appendix. Since the inter-wire capacitance $C$ and inductance $L$ both remain constant along the wires, the Maxwell equations reduce to an ordinary differential equation (2).

The electric field $\mathbf{E}=-\nabla \phi+i k \mathbf{A}$ is calculated from the potentials (10) and (11) as

$$
\begin{aligned}
E_{x} & =-\frac{2 Q_{0} d}{(d / 2)^{2}-x^{2}} \sin \left(\frac{G z}{a}\right) \sec (G), \\
E_{z} & =-\frac{2 Q_{0}}{a G} \ln \left|\frac{d / 2+x}{d / 2-x}\right| \times \\
& {\left[G^{2} \cos \left(\frac{G z}{a}\right) \sec (G)-a^{2} k^{2}\left(1-\cos \left(\frac{G z}{a}\right) \sec (G)\right)\right], }
\end{aligned}
$$

where we still assume that $|x| \ll a,|z|<a,|x-d / 2| \gg b$ and $|x+d / 2| \gg$ $b$. The transverse electric field $E_{x}$ changes its sign with the coordinate $z$ vanishing at $z=0$. Yet, on average the ratio $\left|E_{x}\right| /\left|E_{z}\right|$ is estimated near the resonance $(G \approx \pi / 2)$ as $\left|E_{x}\right| /\left|E_{z}\right| \sim a / d \gg 1$, that is the transversal electric field is on average much larger than the longitudinal field at MPR. Near the wires transverse field $E_{x}$ increases even more: $\left|E_{x}\right| \sim Q_{0} / b$. The potential drop $\Delta U$ between the points $x_{1}=d / 2+l / 2$ and $x_{2}=d / 2-l / 2(2 b<l \ll d)$ is estimated from Eq. (13) as $-2 l Q(z) / d$, and the corresponding electric field $E_{\text {out }} \simeq-2 Q(z) / d$. This field should be considered an external field for the wire at the coordinate $y=d / 2$. The internal transverse potential drop across the wire is estimated as $\left|U_{\text {in }}\right| \simeq b\left|E_{\text {out }}\right| /\left|\varepsilon_{m}\right| \simeq 2|Q(z)| b /\left(d\left|\varepsilon_{m}\right|\right)$, where $\varepsilon_{m}$ 
is the metal dielectric constant, which is assumed to be large $\left|\varepsilon_{m}\right| \gg 1$. The problem of the internal transverse field closely resembles the classical problem of the field induced in a dielectric cylinder by another charged cylinder placed parallel to the first cylinder. An elegant solution of the problem can be found in Ref. [25], Sec.7. In the discussed case $\left|\varepsilon_{m}\right|, d / b \gg 1$ it gives the above obtained estimate for $U_{i n}$. The ratio of the potential drop $U_{i n}$ across a wire to the potential drop $U(z)$ between the wires equals to

$$
\left|\frac{U_{\text {in }}(z)}{U(z)}\right| \simeq \frac{b}{d\left|\varepsilon_{m}\right| \ln (d / b)} \ll 1 .
$$

For any practical purpose we can neglect $U_{i n}$ in comparison to $U$, which allows to reduce the problem of charge and current distribution in the two wire system to the solution of the ordinary differential equation (2) for the electric current $I(z)$. Condition (15) is important for the developed analytical theory of MPR in the system of two thin rods. However, we can envision a system (e.g. two closely packed metal nanowires or hemispheres) that still reveals MPR, but condition (15) is not applied.

To clarify the nature of the resonance, it is instructive to compute the ratio of the electric and magnetic energies at the resonance:

$$
\frac{\mathcal{E}_{E}}{\mathcal{E}_{M}} \sim c^{2} \frac{C^{-1} \int|Q(z)|^{2} d z}{L \int|I(z)|^{2} d z} \approx \frac{g^{2}}{k^{2}} \approx 1-\frac{2}{\ln (d / b) k^{2} b^{2}\left|\varepsilon_{m}\right|},
$$

where we assume that the spatial frequency $g$, given by Eq. (3), is close to the resonance $(g a \approx \pi / 2)$ and use the expressions for the specific capacitance $C \simeq[4 \ln (d / b)]^{-1}$ and inductance $L \simeq 4 \ln (d / b)$ derived in the Appendix. In the electrostatic limit $\left|8 C\left[(k b)^{2} \varepsilon_{m}\right]^{-1}\right| \gg 1$ we obtain $U_{E} / U_{M} \gg 1$ thus explaining the name given to this regime. Because of the symmetry of the electric potential considered here, it is clear that such polarization cannot be induced by any uniform electric field. Therefore, the discussed resonance can be classified as the dark mode [30].

The electric current $I(z)$ is found from Eq. (2) and used to calculate the magnetic moment of the wire pair $\mathbf{m}=(2 c)^{-1} \int[\mathbf{r} \times \mathbf{j}(\mathbf{r})] d \mathbf{r}$, where $\mathbf{j}(\mathbf{r})$ is the density of the current and the integration is over the two nanowires. Thus we obtain

$$
m=\frac{1}{2} H_{0} a^{3} \ln (d / b)(k d)^{2} \frac{\tan G-G}{G^{3}} .
$$

The metal permittivity $\varepsilon_{m}$ has a large negative value in the optical/near-IR range while its imaginary part is small; therefore, the magnetic moment $m$ 
has a resonance at $G \approx \pi / 2$ (see Eq. 3 ) when $m$ attains large values. For a typical metal we can use the Drude formula (5) for $\varepsilon_{m}$, where the relaxation parameter is small $\omega_{\tau} / \omega \ll 1$. Then the normalized magnetic polarizability $\alpha_{M}$ has the following form near the MPR:

$$
\alpha_{M}=\frac{4 \pi m}{H_{0} V}=\frac{16 a d \omega_{p}}{\lambda^{2} \omega_{r} \sqrt{2 \ln (d / b)}}\left[1-\omega / \omega_{r}-i \omega_{\tau} /\left(2 \omega_{r}\right)\right]^{-1},
$$

where $V=4 a b d$, and MPR frequency $\omega_{r}=b \pi \omega_{p} \sqrt{2 \ln (d / b)} /(4 a)$. Note that the magnetic moment contains a pre-factor $\omega^{2} d a / c^{2} \ll 1$ that is small in the electrostatic limit valid for sub-wavelength nanostructures, as was predicted earlier [18]. Close to the resonance $(G=\pi / 2)$ the enhancement factor can be very large for optical and infrared frequencies because of the high quality of the plasmon resonance for $\omega_{r} \ll \omega_{p}$. Therefore, the total pre-factor in Eq. (18) can be of the order of one, thereby enabling the excitation of a strong MPR.

Although the electric field energy near resonance is also very high, it is primarily concentrated in the perpendicular to the wires component of the electric field connecting the two wires as it was discussed after Eqs. (12) and (13). If the wavevector of the propagating wave is in the plane of the wires, and perpendicular to the wires, then the described above MPR does not strongly affect the electric field component parallel to the wires. The integral from the electric field, which is generated in magnetic resonance between the wires, exactly equals to zero as it follow from Eqs. (12) and (13). Envisioning a composite material that consists of such wire pairs, we expect that the magnetic plasmon resonance will not contribute to the dielectric permittivity in the direction parallel to the wires. Therefore, such a medium is not bi-anisotropic [24] and can be described by two effective separate parameters: $\varepsilon$ and $\mu$. Prior work on inducing magnetic moments in nanostructured materials $[17,18]$ in the electrostatic regime dealt with highly symmetric (round) nanowires. Here we demonstrate that magnetic moment can also be induced in the strongly non-symmetric (specifically, horseshoe-shaped) nanostructures with a large negative value of $\varepsilon$.

We now consider a metal nanoantenna that has a horseshoe shape, which is obtained from a pair of nanowires by shorting it at one of the ends (see Fig. 2). When the quasi-static condition $\left|8 C\left[(k b)^{2} \varepsilon_{m}\right]^{-1}\right| \gg 1$ holds, the electric current $I(z)$ in a horseshoe nanoantenna can be obtained from Eq. (2), where the boundary condition changes to $I_{z=a}=(d I / d z)_{z=0}=0$ and, as 


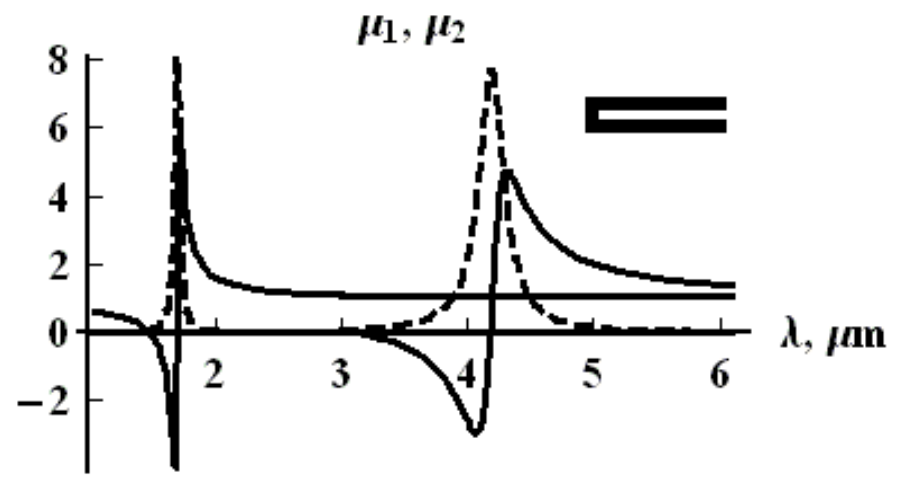

Figure 2: Optical magnetic permeability $\mu=\mu_{1}+\mu_{2}\left(\mu_{1}-\right.$ continous line, $\mu_{2}$-dashed line) estimated from Lorenz-Lorentz formula for the composite containing $\sqsubset$ shaped silver nanoantennas; volume concentration $p=0.3$; left curves: $a=200 \mathrm{~nm}, d=50 \mathrm{~nm}, b=13 \mathrm{~nm}$; right curves $a=600 \mathrm{~nm}$, $d=90 \mathrm{~nm}, b=13 \mathrm{~nm}$; silver dielectric constant is estimated from the Drude formula (5).

above, $a \gg d \gg b$. It is easy to check that the magnetic polarizability $\alpha_{M}$ is still given by Eq. (18), where $a$ is now equal to the total length of the horseshoe nanoantenna. Therefore, the horseshoe nanoantenna provides the same magnetic polarizability $\alpha_{M}$ at the twice shorter length. Magnetic permeability $\mu=\mu_{1}+i \mu_{2}$ for a metamaterial where the silver horseshoe nanoantennas are oriented in one direction (" $z$ " direction in Fig. 1) and are organized in the periodic square lattice is shown in Fig. 2; the optical parameters for silver were taken from [28]. As one can see in the figure, the negative magnetism can be observed, for example, in the near-infrared part of the spectrum, including the telecommunication wavelength of $1.5 \mu \mathrm{m}$.

\section{Numerical Simulations of two-dimensional structures}

To obtain a magnetically active in the optical range material, it might be more convenient to employ (and much easier to model) a "two-dimensional" 


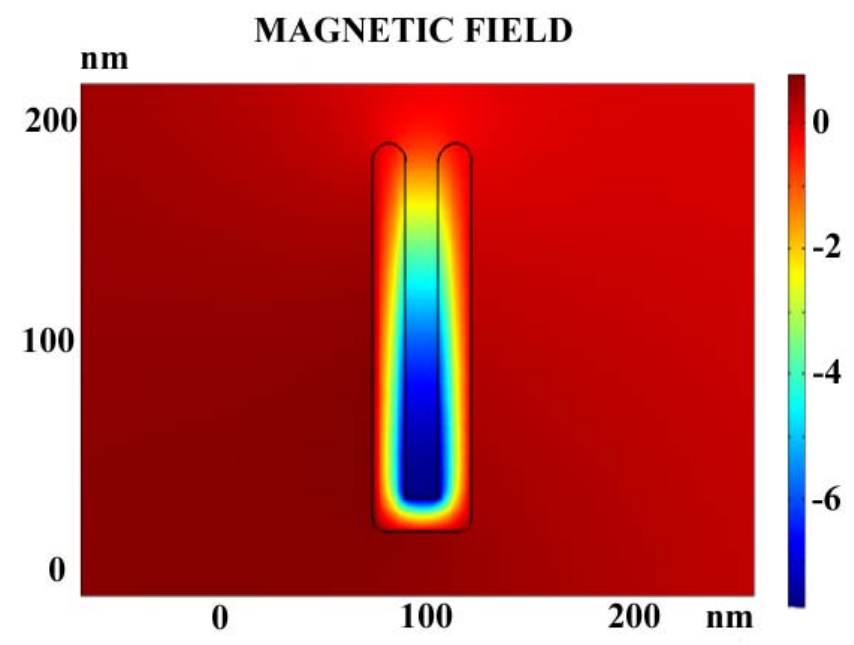

Figure 3: Magnetic plasmon resonance in a silver horseshoe-shaped nanoantenna placed in a maximum of external field $H_{0}$ which is directed perpendicular to the plane; the frequency corresponds to $\lambda=1.5 \mu \mathrm{m}$; silver dielectric constant is estimated from the Drude formula (5).

meta-material, with the nanoantennas having a horseshoe shape in $x, y$ plane and infinitely extended in $z$-direction. When the quasi-static condition $\left|k^{2} b d \varepsilon_{m}\right| \ll 1$ holds ( $b$ and $d$ are thickness and distance between the opposite walls, correspondingly), the MPR frequency $\omega_{r}$ is defined by the equation $G_{2}=2 a \sqrt{-2 /\left(\varepsilon_{m} b d\right)}=\pi / 2$. The resonant magnetic field is shown in Fig. 3. The finite elements code FEMLAB [27] was used to calculate the field distribution. Note that the magnetic field inside the horseshoe is large and of the opposite sign with the external field $H_{0}$, resulting in a negative magnetic permeability in a close proximity to the magnetic plasmon resonance. To estimate the effective magnetic permeability we use an earlier developed approach [13], which gives $\mu_{z}=1+p\left(s H_{0}\right)^{-1} \int\left(H_{\text {in }}-H_{0}\right) d s=$ $(32 / \pi) a^{2} p \lambda^{-2}\left(\pi / 2-G_{2}\right)^{-1}$ for a plasmonic crystal composed by the horseshoe nano-antennas, where $H_{i n}$ is the magnetic field inside a horseshoe, the integration is over the area $s=d a, p$ is the concentration of the nanoantennas organized in a square lattice. For a good optical metal, such as gold or silver, $\mu_{z}$ becomes large and negative for $\omega>\omega_{r}$. For example, the magnetic 


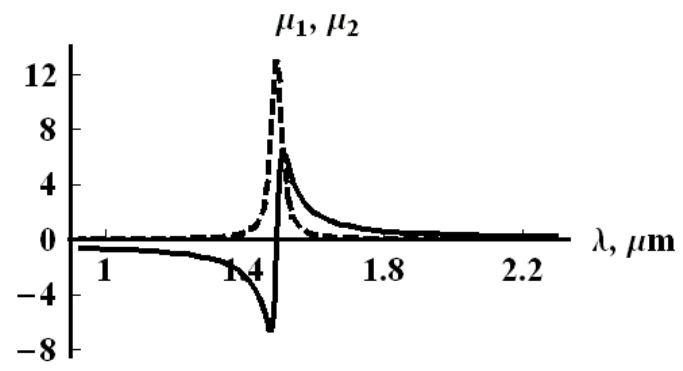

Figure 4: Optical magnetic permeability $\mu=\mu_{1}+i \mu_{2}\left(\mu_{1}-\right.$ continuous line, $\mu_{2}$-dashed line) of the composite containing silver nanoantennas shown in Fig. 3 organized in a square lattice; volume concentration $p=0.4$; silver dielectric constant is estimated from the Drude formula (5).

permeability (both real and imaginary parts) of a metamaterial composed of silver horseshoes has a sharp resonance for $\omega=\omega_{r}$ shown in Fig. 4 . The real part $\mu_{1}$ of the optical magnetic permeability turns negative for $\omega>\omega_{r}$. Figure 4 also reveals the spectral range $\lambda \geq 1 \mu m$ where $\mu_{1}$ is still less than -1 while the relative losses are small: $\delta=\mu_{2} / \mu_{1} \ll 1$. The losses are crucial for a such application of NIM as the perfect lens. Losses could, in principle, be further reduced by cooling the metal nanoantennas to cryogenic temperatures. Simple estimates show that even at the liquid nitrogen temperature the electron mean free path becomes of the order of the horsshoes size. The optical properties of metals are not well understood when the mean free path becomes larger than the nanoantenna size. For these reasons we only consider meta-materials at the room temperature.

To illustrate the effective magnetic properties of the horseshoe metamaterials, we simulated em wave propagation in the plasmonic crystal composed from silver nano-antennas shown in Fig. 3. (The first three horseshoe columns are well seen in Fig. 5a). The em wave is incident on the crystal from the left, its vacuum wavelength is $\lambda=1.4 \mu \mathrm{m}$. The wave is evanescent in the crystal, as can be clearly seen from Fig. 5, and the transmittance $T<10^{-6}$. This evanescence is due to the negative magnetic permeability of the crystal that could be rather large at the resonance (see Fig. 4). One way of making this crystal transparent is to fill the space between the columns of the horseshoes by a material with negative dielectric constant $\varepsilon=-3$. 

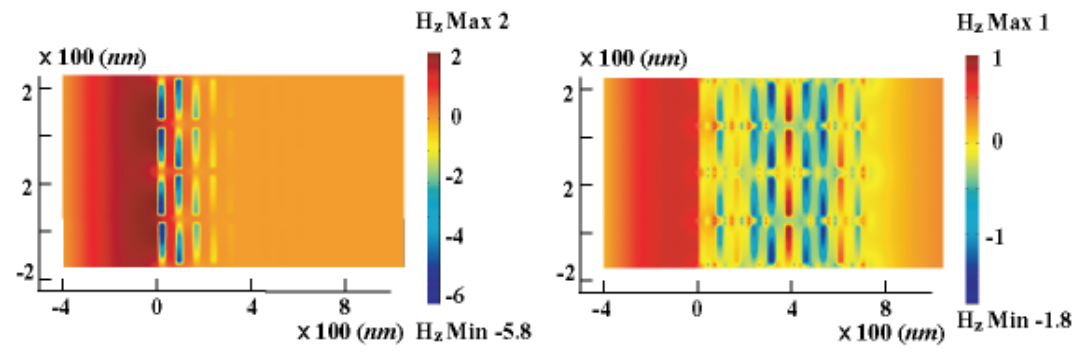

Figure 5: Wave propagation through a two-dimensional (infinitely extended in the normal to the page direction) plasmonic crystal near the plasmon magnetic resonance. The crystal is composed of the horseshoe-shaped silver nano-antennas shown in Fig. 3, volume filling ratio $p=0.4, \lambda=1.4 \mu \mathrm{m}$. Magnetic field $\mathbf{H}$ of the incident em wave is directed along $z$-axis perpendicular to plane of picture; $H_{z}=1$ in the incident wave. (Left) Spaces between the horseshoes are filled with vacuum: no propagation. (Right) Spaces between the horseshoes are filled with a hypothetical $\varepsilon=-3$ material: wave propagates into the structure.

We speculate that such a modification can lead to a double-negative metamaterial. This is confirmed by the numerical simulations. The results are shown in Fig. 5 (right). Indeed, the addition of a negative- $\varepsilon$ material turns our negative- $\mu$ meta-material into transparent NIM. Note that the negative- $\varepsilon$ material was added only between the adjacent columns of horseshoe-shaped nano-antennas. No additional material was placed in the exterior of the nanoantenna. This was done intentionally because modification of the nanostructure region where most of the magnetic field is concentrated is known [29] to affect the magnetic effective permittivity.

Interestingly, the horseshoes themselves can exhibit a double-negative behavior when they are closely packed. We designed a two-dimensional dense periodic structure consisting of alternating up and down horseshoe nanoantennas. One half of the elementary cell is shown in Fig. 6a; another half of the elementary sell is obtained by $180^{\circ}$ degree rotation in $x y$ plane. (The structure then repeats itself in $x$ and $y$ directions; separation between antenna centers is $80 \mathrm{~nm}$, horizontal periodicity is $160 \mathrm{~nm}$, vertical periodicity is 400 $\mathrm{nm}$; see Fig. 5). Dispersion relation $\omega\left(k_{x}\right)$ for the electromagnetic wave propagating through the periodic structure in $x$ direction has been calculated 
by numerically solving the Maxwell's equation for magnetic field $H_{z}$. For computational simplicity, we have assumed a hypothetical lossless plasmonic material with the frequency-dependent dielectric permittivity $\varepsilon=1-\omega_{p}^{2} / \omega^{2}$, where $2 \pi c / \omega_{p}=225 \mathrm{~nm}$. The frequency $\omega$ and the wavevector $k$ are normalized to $\omega_{0}=2 \pi c / \lambda_{0}$ and $k_{0}=2 \pi / \lambda_{0}$, respectively, where $\lambda_{0}=1.5 \mu \mathrm{m}$.

Remarkably, one of the propagating modes (shown in Fig. 6b) exhibits the left-handedness: its group velocity $v_{g r}=\partial \omega / \partial k$ opposes its phase velocity. Fig. 6a shows the magnetic field profile and the electric field inside the elementary cell for $k_{x}=0$ (magnetic cutoff condition corresponding to $\mu=0$ ). Magnetic field is concentrated inside the horseshoes, and has opposite signs in the adjacent horseshoes. The dominant field in the structure is $E_{x}$ which does not contribute to the Poynting flux in the propagation direction. Note that arrows in Fig. 6 indicate the value the electric field takes at their origin. Then Fig. 6 clearly indicates that the electric field of MPR is mainly confined inside the horseshoes and is almost negligible in the metal. As we have mentioned above there is a half of the elementary cell in Fig:6. In another half cell the $E_{x}$ field has opposite direction so that the average $E_{x}$ is zero. The electric field is primarily potential (i.e. can be derived from an electrostatic potential), but has a non-vanishing solenoidal component that produces the magnetic field. The potential drop between the metal arms is much larger than the potential drop between external and internal interfaces of an arm. This behavior of the MPR electric field, obtained from direct computer simulations, resembles our analytical results for two wire system (see discussion at Eqs. (12) and (13)). The fact that the dominant electric field $E_{x}$ does not change the sign inside the cell along the direction of the propagation indicates that the mode in question does not owe its negative dispersion to the band-folding effect common in photonic crystals. The lefthanded behavior occurs in the vicinity of $\lambda=1.88 \mu \mathrm{m}$, which is close to the $\mathrm{MPR}$ resonance. The negative $\varepsilon$ necessary for the negative refractive index is induced by the proximity of the dipole-type electrostatic resonances [17].

\section{Conclusions}

In conclusion, a new phenomenon of a magnetic plasmon resonance in metallic horseshoe-shaped split rings was described. This resonance is distinctly different from the geometric LC resonance described earlier for split rings because it is determined by the plasmonic properties of the metal. This 

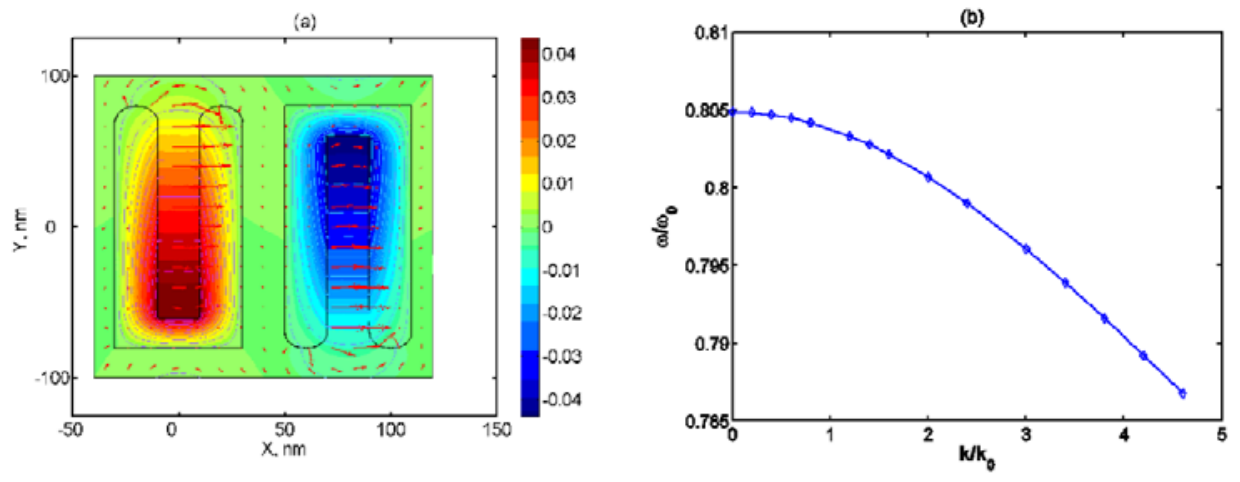

Figure 6: Plasmonic crystal composed from the horseshoe-shaped metal nanoantennas; separation between antenna centers is $80 \mathrm{~nm}$. Magnetic (color and contours) and electric (arrows) fields inside a periodic array of horseshoeshaped nano-antennas at the cutoff $\left(k_{x}=0\right)$. (b) Dispersion relation $\omega$ v. s. $k_{x}$ for a left-handed electromagnetic wave.

work paves the way to designing metallic meta-materials that are magnetically active in the optical and near-infrared spectral ranges. Presented threedimensional analytic calculations and two-dimensional numerical simulations reveal that resonantly enhanced magnetic moments can be induced in very thin (thinner than a skin depth) split rings with typical dimensions much shorter than the wavelength (on the order of 100 nanometers). Periodic arrays of such horseshoe-shaped nanoantennas can be used to design lefthanded meta-materials by exploiting the proximity of electric resonances in the dielectric permittivity $\varepsilon$ and magnetic permeability $\mu$.

Acknowledgments: The authors acknowledge useful contributions and discussions with D. Genov, and V. Podolskiy, and G. Tartakovsky. This work was supported in part by the NSF grants ECS-0210445 and DMR-0121814 and by the ARO MURI grant W911NF-04-01-0203.

\section{Appendix}

We derive equations for the capacitance $C$ and inductance $L$ between two parallel wires of the radius $b$ and length $a$ separated by the distance $d$. We 
suppose from the beginning that $b \ll d$ and $d \ll a$. We also suppose that the dielectric constant $\varepsilon_{m}$ of the wires is large in absolute value $\left|\varepsilon_{m}\right| \gg 1$ whereas the skin depth $\delta \sim k \sqrt{\left|\varepsilon_{m}\right|} \ll b$ as it is explained in the text. To find the capacitance $C$ we first calculate the electric potential $\Phi_{a}$ in the point with coordinate $\mathbf{r}_{a}$ at the surface of the wire (point $a$ in Fig. 1) obtaining

$$
\Phi_{a}=\int q_{1}\left(\mathbf{r}_{1}\right) \frac{\exp \left(i k r_{a 1}\right)}{r_{a 1}} d \mathbf{r}_{1}+\int q_{2}\left(\mathbf{r}_{2}\right) \frac{\exp \left(i k r_{a 2}\right)}{r_{a 2}} d \mathbf{r}_{2}
$$

where $r_{a 1}=\left|\mathbf{r}_{a}-\mathbf{r}_{1}\right|, r_{a 2}=\left|\mathbf{r}_{a}-\mathbf{r}_{2}\right|, q_{1}$ and $q_{2}$ are the electric charges distributed over the surface of the rods; the integration goes over the surface of the first $(a, d)$ and second $(b, c)$ rods in Fig. 1. For further consideration we choose the coordinate system $\{x, y, z\}$ with $z$ axis along the $(a, d) \operatorname{rod}$, origin in the center of the system and the $x$ axis connecting the axes of the rods so that $y$ axis is perpendicular to the plane of two rods. We introduce the vector $\mathbf{d}=\{d, 0,0\}$ between the wires and two dimensional unit vector $\rho(\phi)=\{\cos \phi, \sin \phi\}$ in $\{x, y\}$ plane, where $\phi$ is the polar angle. Then the vectors in Eq. (19) can be written as $\mathbf{r}_{a}\left(\phi_{a}, z_{a}\right)=\left\{b \rho\left(\phi_{a}\right)+\mathbf{d} / \mathbf{2}, z_{a}\right\}$, $\mathbf{r}_{1}\left(\phi_{1}, z_{1}\right)=\left\{b \rho\left(\phi_{1}\right)+\mathbf{d} / \mathbf{2}, z_{1}\right\}$, and $\mathbf{r}_{2}\left(\phi_{2}, z_{2}\right)=\left\{b \rho\left(\phi_{2}\right)-\mathbf{d} / \mathbf{2}, z_{2}\right\}$. It follows from the symmetry of the problem that the electric charge $q_{1}(\phi, z)=$ $-q_{2}(\phi+\pi, z)$ (recall that we consider antisymmetric mode when the electric currents in the rods are equal in absolute values but follows in the opposite directions.) We rewrite Eq. (19) splitting it in two parts

$$
\begin{gathered}
\Phi_{a} \equiv \Phi_{a}^{(0)}+\Phi_{a}^{(1)} \\
\Phi_{a}^{(0)}\left(z_{a}, \phi_{a}\right)=\int_{\phi=0}^{\pi} \int_{z=-a}^{a} q(\phi, z)\left[\frac{1}{\sqrt{\Delta z^{2}+b^{2} \Delta \rho_{1}^{2}}}-\right. \\
\left.\frac{1}{\sqrt{\Delta z^{2}+\left(b \Delta \rho_{2}-\mathbf{d}\right)^{2}}}\right] d z b d \phi, \\
\Phi_{a}^{(1)} \equiv \int q\left(\mathbf{r}_{1}\right) \frac{\exp \left(i k r_{a 1}\right)-1}{r_{a 1}} d \mathbf{r}_{1}-\int q\left(\mathbf{r}_{2}\right) \frac{\exp \left(i k r_{a 2}\right)-1}{r_{a 2}} d \mathbf{r}_{2},
\end{gathered}
$$

where $\Delta z=z_{a}-z, \Delta \rho_{1}=\rho_{a}-\rho=\left\{\cos \phi_{a}-\cos \phi, \sin \phi_{a}-\sin \phi\right\}, \Delta \rho_{2}=\rho_{a}+$ $\rho=\left\{\cos \phi_{a}+\cos \phi, \sin \phi_{a}+\sin \phi\right\}$. Note that dimensionless vectors $\Delta \rho_{1}$ and 
$\Delta \rho_{2}$ satisfy $\left|\Delta \rho_{1}\right|,\left|\Delta \rho_{2}\right|<2$ so that the the second terms in the radicals in Eq. (21) are much less than $a$.

The electric currents in the rods and, correspondingly, electric charge $q$ changes with coordinate $z$ on the scale $\sim a$ which is much larger than the distance $d$ between the rods. Therefore we can neglect $z$ variation of the electric charge for $|\Delta z|<d$. On the other hand the term in the square brackets in Eq. (21) vanishes as $\sim d^{2} /|\Delta z|^{3}$ for $|\Delta z|>d$. This allows to replace in Eq. (21) the charge $q(z, \phi)$ by its value $q\left(z_{a}, \phi_{a}\right)$ in the observation point $\mathbf{r}_{a}$ obtaining

$$
\begin{aligned}
\Phi_{a}^{(0)}\left(z_{a}, \phi_{a}\right) & =\int_{\phi=0}^{2 \pi} q\left(z_{a}, \phi\right) \int_{z=-a}^{a}\left[\frac{1}{\sqrt{\Delta z^{2}+b^{2} \Delta \rho_{1}^{2}}}-\right. \\
& \left.\frac{1}{\sqrt{\Delta z^{2}+\left(b \Delta \rho_{2}-\mathbf{d}\right)^{2}}}\right] d z b d \phi ;
\end{aligned}
$$

the accuracy of this replacement is about $(d / a)^{2} \ll 1$. Since we consider the quasistatic limit when the distance between the rods $d \ll \lambda$ and the metal dielectric constant $\left|\varepsilon_{m}\right| \gg 1$ the potential lines in $\{x, y\}$ plane are close to the static case. Therefore we can safety suppose that the angle distribution of the electric charge $q(z, \phi)$ is the same as it would be in the case of two infinite metal cylinders in the static case: $q(z, \phi)=$ $Q(z) \sqrt{(d / b)^{2}-4} /(2 \pi(d+2 b \cos \phi))$, where $Q(z)$ is the electric charge per unit length of the rod so that $\int_{\phi=0}^{\phi=2 \pi} q(z, \phi) b d \phi=Q(z)$. Then the integral in Eq. (24) gives the

$$
\Phi_{a}^{(0)}(z)=Q(z) \operatorname{arccosh}\left(\frac{d^{2}}{2 b^{2}}-1\right)+O\left((d / a)^{2}\right),
$$

where the second term includes all corrections to the integral (21) due to finite size of the system. For the thin wires, considered here, when the radius $b$ is much smaller than the distance $d$ between the wires the potential $\Phi_{a}^{(0)}$ approximates as

$$
\Phi_{a}^{(0)}(z) \simeq Q(z) 2[\ln (d / b)] .
$$

The second term $\Phi_{a}^{(1)}$ in Eq. $(20)$ is small in the limit of $a \ll \lambda$, i.e., $k r_{a 1}, k r_{a 2} \ll 1$. The real part of $\Phi_{a}^{(1)}$ gives a small correction $\sim(d / a)^{2}$ to the 
potential $\Phi_{a}^{(0)}$ that can be neglect. The imaginary part is important regardless of its absolute value since it gives so-called radiative losses. To estimate the losses we assume that $b / d \ll 1$ and neglect the angle dependence of the charge distribution. Then we expand Eq. (23) in series of $k$ and linearly approximate $Q(z) \simeq q_{1} z$ (recall that $Q(z)$ is an odd function of $z$ ) obtaining

$$
\Phi_{a}^{(1)}(z) \simeq-i Q(z)(a k)^{3}(k d)^{2} / 45,
$$

where we neglect the terms with higher orders on $k$ as well as all terms on the order of $(b k)^{2}$.

Due to the symmetry of the system the potential difference $U=\Phi_{a}-$ $\Phi_{b}$ between points $a$ and $b$ (see Fig. $1 ; z_{a}=z_{b}$ ) equals to $U=2 \Phi_{a}$. The capacitance $C$ defined as $C=Q(z) / U(z)$ is given by

$$
\begin{gathered}
\frac{1}{C} \simeq 2 \operatorname{arccosh}\left[(d / b)^{2} / 2-1\right]-i \frac{2}{45}(a k)^{3}(k d)^{2} \simeq \\
4 \ln \left(\frac{d}{b}\right)-i \frac{2}{45}(a k)^{3}(k d)^{2},
\end{gathered}
$$

where the first term is the capacitance between two parallel infinite cylinders (see [25] Ch. 3 ); the second term gives the radiative losses due to the retardation effects.

Consider now the inductance $L$ between the wires. To find the inductance $L$ we first calculate the vector potential $A_{a}$ in the point with coordinate $\mathbf{r}_{a}$ inside the wire. We neglect the edge effects and assume that the vector potential is parallel to the axes of the wires obtaining

$$
A_{a}=\frac{1}{c} \int j(\mathbf{r})\left(\frac{\exp \left(i k r_{a 1}\right)}{r_{a 1}}-\frac{\exp \left(i k r_{a 2}\right)}{r_{a 2}}\right) d \mathbf{r}
$$

where $r_{a 1}=\left|\mathbf{r}_{a}-\mathbf{r}\right|$ and $r_{a 2}=\left|\mathbf{r}_{a}-\mathbf{r}+\mathbf{d}\right|, j\left(\mathbf{r}_{1}\right)$ is the density of the current and the integration goes over the volume of the first wire. We consider the quasistatic case when the skin effect is small $\left(k b \sqrt{\left|\varepsilon_{m}\right|} \ll 1\right)$. Then the electric current uniformly distributes over the cross-section of a wire and $j(\mathbf{r})=I(z) /\left(\pi b^{2}\right)$. Following the procedure used above for calculating the electric potential, the vector potential is expressed as $A_{a}=A_{a}^{(0)}+A_{a}^{(1)}$, where

$$
A_{a}^{(0)}=\frac{1}{c} \int \frac{I(z)}{\pi b^{2}}\left(\frac{1}{r_{a 1}}-\frac{1}{r_{a 2}}\right) d \mathbf{r}
$$




$$
A_{a}^{(1)}=\int \frac{I(z)}{\pi b^{2}}\left(\frac{\exp \left(i k r_{a 1}\right)-1}{r_{a 1}}-\frac{\exp \left(i k r_{a 2}\right)-1}{r_{a 2}}\right) d \mathbf{r} .
$$

The term $A_{a}^{(0)}$ estimates in the same way as $\Phi_{a}^{(0)}$. As result we obtain the vector potential $A_{a}^{(0)}$ averaged over the cross-section of the wire in the following form

$$
A_{a}^{(0)}(z) \simeq \frac{I(z)}{2 c}\left[4 \ln \left(\frac{d}{b}\right)+1\right],
$$

where $I(z)$ is electric current, and we neglect terms on the order $(b / d)^{2} \ll 1$ and $(d / a)^{2} \ll 1$. To estimate Eq. (33) we expand it in series on $k$ obtaining that the linear term equals to zero, $k^{2}$ term gives small correction $\left(\sim(k d)^{2}\right)$ to $A_{a}^{(0)}$ and the third order on $k$ gives the radiative losses, namely

$$
A_{a}^{(1)} \simeq i(k d)^{2} k \frac{1}{c} \int I(z) d z \sim 2 i \frac{I(z)}{c}(k d)^{2} k a,
$$

where we rather arbitrary neglect variation of the current over the rod length in transition to the second estimate. We obtain inductance $L$ form the equation $A_{a}-A_{b}=2 A_{a}=(L / c) I(z)$ as

$$
L=4 \ln \left(\frac{d}{b}\right)+1+4 i(k d)^{2} k a .
$$

The first two terms correspond to the self-inductance per unit length of a system of two parallel infinite wires ([25], Ch.34.) This estimate as well as Eq.(30) are certainly invalid near the ends of the rods, but in calculating the current distribution $I(z)$ and magnetic moment this region is unimportant.

We are now in a position to compare the radiation losses (given by imaginary parts of capacitance $C$ and inductance $L$ ) and the ohmic loss in the metal wires. In near infrared spectral region the dielectric constant $\varepsilon_{m}$ for a "good" optical meal $(A g, A u$, etc.) can be estimated from the Drude formula $(5)$ as $\varepsilon_{m}(\omega) \sim\left(\omega_{p} / \omega\right)^{2}\left(1-i \omega_{\tau} / \omega\right)^{-1}$, where $\omega_{p}$ is plasma frequency and $\omega_{\tau} \ll \omega \ll \omega_{p}$ is the relaxation rate. Thus we obtain that the real part of the rod resistance $R_{\text {ohm }} \sim 8\left(\omega_{\tau} / \omega_{p}^{2}\right)\left(a / b^{2}\right)$ should be compared with "radiation" resistance $R_{\text {rad }} \sim(k d)^{2}(k a)^{2} / c$. For the silver nanowires, considered in the paper, the ohmic losses either larger $\left(R_{o h m}>R_{\text {rad }}\right)$ or much larger $\left(R_{\text {ohm }} \gg R_{\text {rad }}\right)$ than the radiation losses. Therefore we can neglect the imaginary parts of the capacitance $C$ and inductance $L$ and approximate them for simplicity as

$$
L \simeq \frac{1}{C} \simeq 4 \ln \frac{d}{b} .
$$


This estimate is of logarithmic accuracy; its relative error is on the order of $(4 \ln d / b)^{-1}$. Note that the radiation losses crucially depend on the parameter $k a$. Magnetic plasmon resonance address in this paper becomes very broad when $k a>1$, placing a rather sever constraint on the length $2 a$ of the wire.

\section{References}

[1] J. B. Pendry, A. J. Holden, W. J. Stewart, and I. Youngs, Phys. Rev. Lett. 76, 4773 (1996).

[2] A. N. Lagarkov, A. K. Sarychev, Y. R. Smychkovich, and A. P. Vinogradov, J. Elect. Waves and Appl. 6, 1159 (1992); A. N. Lagarkov and A. K. Sarychev, Phys. Rev. B 53, 6318 (1996);

[3] J.B. Pendry, A.J. Holden, D.J. Robbins, W.J. Stewart, IEEE Trans. Microw. Theory 47, 2075 (1999); M.C.K. Wiltshire, J.V. Hajnal, J.B. Pendry, D.J. Edwards, C.J. Stevens, Optic Express 11, 709 (2003).

[4] V. G. Veselago, Soviet Physics Uspekhi 10, 509 (1968).

[5] D.R. Smith, W.J. Padilla, D.C. Vier, S.C. Nemat-Nasser, S. Schultz, Phys. Rev. Lett. 84, 4184 (2000).

[6] J.B. Pendry, Phys. Rev. Lett. 85, 3966 (2000).

[7] For recent references see the special issue of Optics Express 11, No 7 (2003).

[8] A.A. Houck, J.B. Brock, and I.L. Chuang, Phys. Rev. Lett. 90, 137401 (2003).

[9] C.G. Parazzoli, R.B. Greegor, K. Li, B.E.C. Koltenbah, and M. Tanielian, Phys. Rev. Lett. 90, 107401 (2003).

[10] A. Alu, N. Engheta, IEEE T. Microw. Theory 52, 199 (2004).

[11] A.N. Lagarkov, V.N. Kissel, Phys. Rev. Lett. 92, 077401-1 (2004).

[12] S. Linden, C. Enkrich, M. Wegener, J. Zhou, T. Koschny, C.M. Soukoulis, Science 306, 1351 (2004); N. Katsarakis, T. Koschny, M. Kafesaki, E. N. Economy, C. M. Soukoulis, Appl. Phys. Lett. 84, 2943 (2004). 
[13] A.K. Sarychev and V.M. Shalaev, Phys. Rep. 333, 275 (2000).

[14] V. M. Shalaev, W. Cai, U. Chettiar, H.-K. Yuan, A. K. Sarychev, V. P. Drachev, and A. V. Kildishev, arXiv:physics/0504091, Apr. 13, (2005); Laser Phys. Lett. 3, 49-55 (2006); Opt. Lett. 30, 3356-3358 (2005).

[15] V.A. Podolskiy, A.K. Sarychev, and V.M. Shalaev, J. Nonlin. Opt. Phys. Mat. 11, 65 (2002); Optics Express 11, 735 (2003); A.K. Sarychev, V.P. Drachev, H.-K, Yuan, V.A. Podolskiy, and V.M. Shalaev, SPIE Proceedings, 5219, 92, San Diego (2003).

[16] G. Shvets, Phys. Rev. B 67, 035109 (2003).

[17] G. Shvets and Ya. A. Urzhumov, Phys. Rev. Lett. 93, 243902 (2004).

[18] G. Shvets and Ya. A. Urzhumov, J. Opt. A: Pure Appl. Opt. 7, S23 (2005).

[19] G. Shvets and Y. A. Urzhumov, "Negative index meta-materials based on two-dimensional metallic structures", J. Opt. A: Pure Appl. Opt. 7 (2005) (in press).

[20] A.N. Grigorenko, A.K. Geim, H.F. Gleeson, Y. Zhang, A.A. Firsov, I.Y. Khrushchev, and J. Petrovic, Nature, 438, 335 (2005)

[21] A.K. Sarychev and V.M. Shalaev, SPIE Proceedings, 5508, 128 (2004).

[22] C. Enkrich, M. Wegener, S. Linden, S. Burger, L. Zschiedrich, F. Schmidt, J. Zhou, Th. Koschny, C. M. Soukoulis, arXiv:condmat/0504774 v.1, 29 Aprl 2005.

[23] T. J. Yen, W. J. Padilla, N. Fang, D. C. Vier, D. R. Smith, J. B. Pendry, D. N. Basov, and X. Zhang, Science 203, 1494 (2004).

[24] R. Marques, F. Medina, and R. Rafii-El-Idrissi, Phys. Rev. B 65, 144440 (2002).

[25] D. Landau and E.M. Lifshitz, Electrodynamics of Continuous Media, 2nd ed. (Pergamon, Oxford, 1984).

[26] L.V. Panina, A.N. Grigorenko, D.P. Makhnovskiy, Phys. Rev. B 66, 155411 (2002). 
[27] FEMLAB Reference Manual 2003 Version 2.3 Comsol AB, Sweden.

[28] U. Kreibig and M. Vollmer, Optical Properties of Metal Clusters, Springer-Verlag, Berlin, 1995; P.B. Johnson and R.W. Christy, Phys. Rev. B 6, 4370 (1972).

[29] A. L. Pokrovsky and A. L. Efros, Phys. Rev. Lett. 89, 093901 (2002).

[30] M.I. Stockman, S.V. Faleev, and D.J. Bergman, Phys. Rev. Lett. 87, 167401 (2001). 